\title{
Multi-Unit Feature Level Fusion Approach Using PPCA
}

\author{
A. Alice Nithya, C. Lakshmi, Jitesh Anand and Nitish Sekar \\ School of Computing, SRM University, Chennai - 603203, Tamil Nadu, India; \\ alicenithya.a@ktr.srmuniv.ac.in, lakshmi.c@ktr.srmuniv.ac.in, jitesh723@gmail.com,nsekar15@gmail.com
}

\begin{abstract}
Iris recognition system is considered to be one of the most reliable, accurate and stable security systems providing identification or authentication of an individual. In this paper, a multi-unit feature level fusion approach for iris based biometric system is proposed with the aim of improving the recognition accuracy even for inaccurately segmented iris images. From the existing analysis it is clear that for an iris based biometric system to become reliable and accurate, more emphasis should be given to the preprocessing and segmentation stage. In this work Daugman's Integro-Differential Operator is applied on eye images taken in unconstrained situation, and iris region of interest is extracted without eliminating noise factors like eyelid and eyelash occlusions, specular reflections, non-circularity of iris, etc. A novel feature selection technique, Probabilistic Principal Component Analysis (PPCA) is applied to provide good recognition rate by working on missing values of inaccurately segmented iris images. Mean method of multi-unit feature level fusion methodology is proposed to improve feature selection accuracy. The proposed methodology is found to provide better recognition rate of $\mathbf{8 3 . 3 \%}$ even for inaccurately segmented iris images, when experimented on MultiMedia University (MMU) dataset.
\end{abstract}

Keywords: Feature Level Fusion, Feature Selection, Multi-unit Biometric Fusion, Probabilistic Principal Component Analysis (PPCA), Recognition Rate

\section{Introduction}

Biometrics refers to the utilization of certain traits or characteristics of a person for the purpose of identification ${ }^{1}$. These traits can be either physical or behavioral. A few examples of such traits are iris, retina, DNA, fingerprint, voice, gait, etc. ${ }^{2}$. The unique features that are obtained from these biometric traits are ideal for security systems because they can't be stolen or duplicated, as opposed to a key or a password used in traditional security systems ${ }^{1,2}$. Iris recognition is one of the most reliable, and promising option amongst many other biometric systems because the iris is unique - the patterns formed by arching ligaments, ridges, colours, freckles etc. are different for each and every person - and immutable - the iris of a person remains unchanged throughout his or her lifetime ${ }^{3,4}$. Even the left and right irises of a person have different patterns. Also, iris as a biometric modality is more measurable as compared to fingerprints, i.e. images of the iris can be easily acquired without direct contact with the user, which makes it the better option. Thus, iris recognition can be ideal for the purpose of identification in various fields such as personal authentication, banking, law enforcement, surveillance, etc ${ }^{4}$.

Iris recognition systems or any biometric system in general, can be implemented using one of the following approaches $^{5,6}$ :

${ }^{*}$ Author for correspondence 
1. Unimodal - A biometric system using a single biometric trait. These systems face certain limitations like sensor data noise, spoof attacks, etc. But, such systems face certain limitations, such as sensor data noise, spoof attacks etc., which lead to a reduction in accuracy and performance. Such issues can be reduced by following a multi modal approach.

2. Multimodal - A biometric system using more than one biometric trait collected from different sources.

3. Multi-unit - Multiple units of same modality are fused and processed further.

The basic operations performed in an iris recognition system are as follows:

1. Image acquisition, i.e. capturing the image;

2. Iris Boundary Localization: In this step the pupiliris inner boundary and iris-sclera outer boundary are identified to localize the region of interest ;
3. Normalization: Circular iris ring is unwrapped to a rectangular structure;

4. Feature extraction: From the rectangular structure features are extracted and a template is generated and stored during enrolment stage;

5. Matching: During identification/ authentication stage, identified feature vector is compared against the stored templates and a match/ no match is found.

In this paper, we propose a multi-unit feature level fusion approach to recognize iris of an individual, i.e. information obtained from the left and right irises of a person are fused together, in order to achieve better performance. We are performing feature level fusion to overcome sensor noises and spoofing attacks. The fusion technique used is the mean method of fusion. Detailed work flow of proposed iris recognition process is depicted in Figure 1.

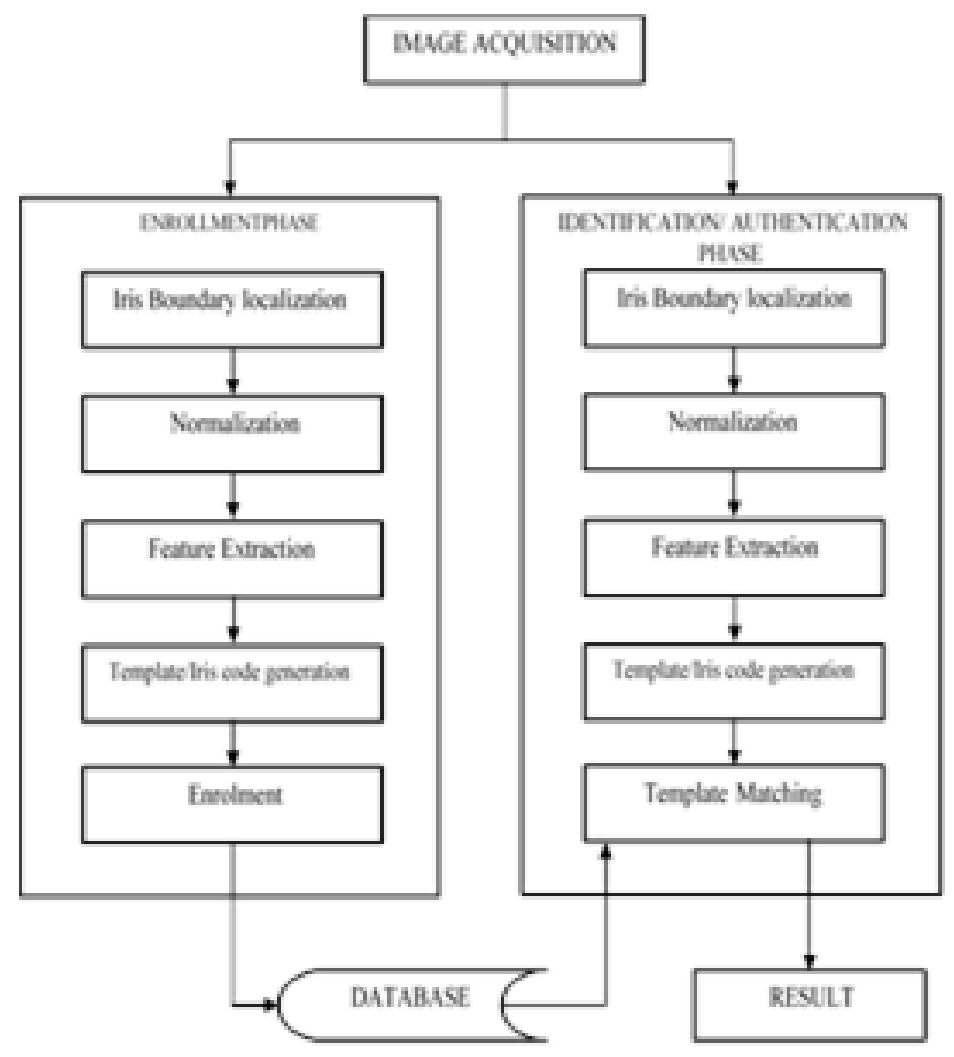

Figure 1. Iris recognition process. 
The paper has been structured as follows:

Section 2 gives an overview of iris segmentation and normalization process. Section 3 explains the implementation of Probabilistic Principal Component Analysis (PPCA) for feature selection. Section 4 provides an overview of the experimental methods and the results obtained through the proposed approach. The final section provides the conclusion.

\section{Iris Segmentation and Normalization}

The first step to be taken, once the image has been acquired, is to pre-process it, i.e. segment and normalize it. Segmentation localizes the region of interest, i.e., iris area and removes the other unwanted portions of an eye. Normalization is performed to convert the segmented image of an iris into a rectangular image, to compensate for the scaling, rotational and translational invariance's, that can be caused due to factors such as changes in the distance from the camera, the changes in the illumination which causes the pupil to dilate or contract, the person's head rotation or the camera tilt, etc.

In our approach, segmentation is performed using Daugman's classical Integro-differential operator ${ }^{7}$, and normalization is performed using Daugman's rubber sheet model $^{7,8}$. One of the major issues in using classical Integro-differential operator is the decrease in performance when implemented on images taken under non-ideal situations like on-the-move, at a distance or non-cooperative setups. Many researchers are working on developing a robust segmentation algorithm to increase the overall system performance, whereas in this work, we tried to use the classical operator and implemented a feature selection algorithm called Probabilistic Principal Component Analysis (PPCA $)^{9}$, which will deal with missing values present in the segmented and normalized image.

\section{Probabilistic Principal Component Analysis}

Probabilistic PCA was developed in, based on probabilistic formulation of PCA, which acts like a good foundation for dealing with missing data values ${ }^{9}$. PPCA can be used in modeling class-conditional densities and hence could be applied for classification problems. PPCA facilitates statistical testing and permits Bayesian methods application. PPCA extends the scope of PCA by estimating the principal components even if the input data has some missing values. PPCA basically seeks a relationship between a $m-b y$ - $n$ dimensional observation data $y$ to its corresponding $k$-dimensional latent (or unobserved) variable vector, $x$, which is normal with mean zero and covariance $\mathrm{I}(k)$. The relationship is:

$$
\mathbf{y} \mathbf{T}=\mathbf{W} * \mathbf{x} \mathbf{T}+\mu+\varepsilon
$$

Where, $y$ is the m-by-n-matrix of observed variable, $\mathrm{x}$ is the row vector of latent variables, and eis an isotropic error term. eis Gaussian with mean zero and covariance of $v * I(k)$, where $v$ is the residual variance ${ }^{10}$. In this case, the value of $\mathrm{k}$ should be less than the rank of the residual variance, but should be greater than 0 .

Here, $W$ relates the latent and observation variables, and the vector $\mu$ permits the model to have a mean with non-zero value. In PPCA, whether a data value is missing or not does not depend on the latent variable given the observed data values, meaning it assume through the input data that the values are missing at random.

Under this PPCA model,

$$
\mathbf{y} \sim \mathrm{N}(\boldsymbol{\mu}, \mathbf{W} * \mathbf{W T}+\mathbf{v} * \mathbf{I}(\mathrm{k}))
$$

For $\mathrm{W}$ and $\mathrm{v}$ there is no closed form of analytical solution available. Hence an Expectation-Maximization (EM) algorithm is used to determine the estimate values by iterative maximization of the corresponding log likelihood. This EM algorithm helps in handling the missing values present in the input data by treating them as additional latent variables. At convergence, the columns of $W$ span the subspace, but they are not orthonormal. PPCA obtains the orthonormal coefficients, for the components by orthogonalization of $\mathrm{W}^{9-11}$. PPCA is considered as a probabilistic version of PCA as the solution in this case is obtained by maximizing a probability.

\section{Experimental Methods and Results}

Non-ideal images taken from MultiMedia University (MMU) dataset ${ }^{12}$, having 450 images, with 5 images per 


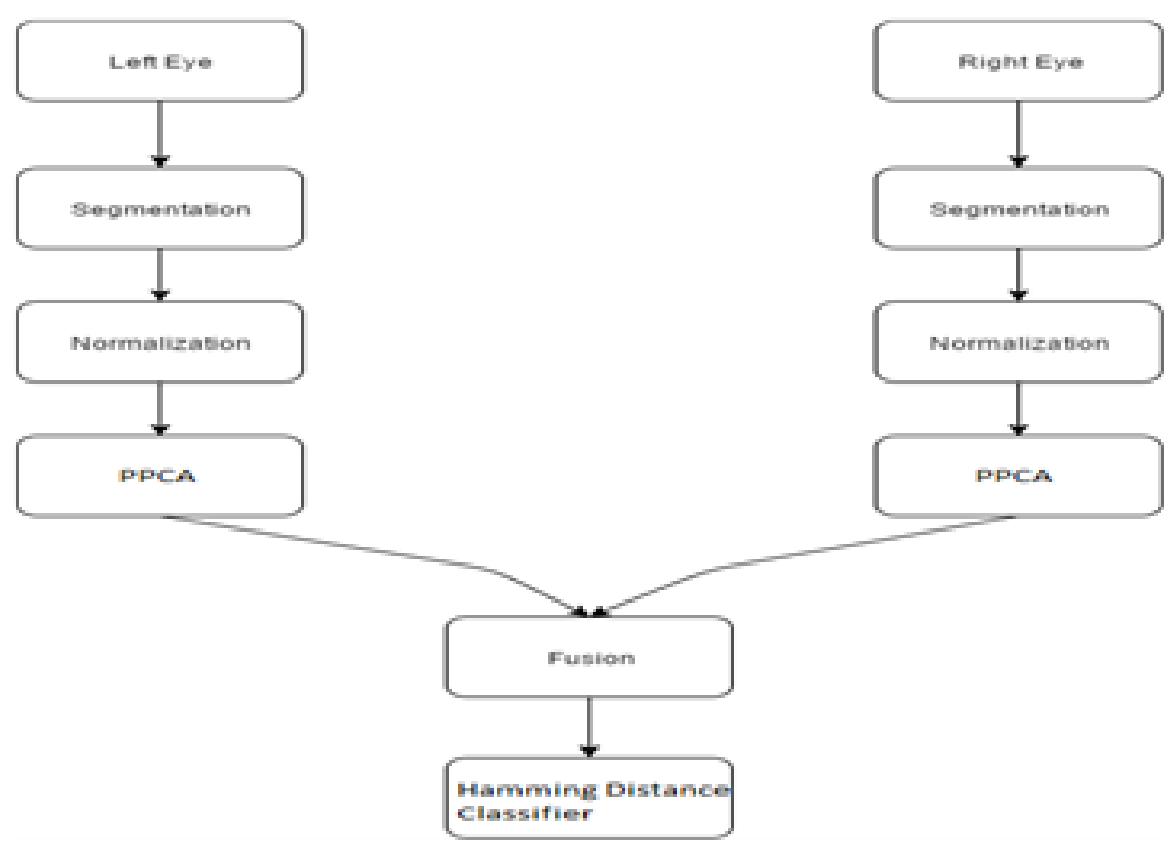

Figure 2. Proposed approach.

iris and 2 irises per subject have been used to test our results in this approach. The result of this work is analyzed with 108 images, i.e. 3 images per iris per subject for 18 subjects.

During enrolment phase, segmentation, normalization and feature selection processes have been executed for both left and right iris of a subject sequentially. Using mean method of fusion generated templates are then fused at feature level and stored in a dataset. During identification/authentication phase, above mention processes are executed and the newly generated fused template is matched against the stored templates for a match/ no match and a decision is made based on the defined threshold value.A generic flowchart of the multi-unit feature level fusion approach using PPCA is shown in Figure 2.

Segmentation and normalization results obtained using Daugman's Integro-differential operator and the rubber sheet normalization model is shown in Figure 3. Normally, biometric systems provide better performance accuracy with accurate segmented results. From Figure3, it has been observed that when Interpolated Differential Operator (IDO) is applied on non-ideal images, the resulting segmented image is not accurate. But, here we have implemented a novel feature selection approach, PPCA, to deal with the missing values present in the inaccurately segmented iris images, occurred due to eyelid and eyelash occlusion, specular reflections, non-circularity of iris, etc. are taken care. This improves the system performance in terms of both accuracy and robustness.

Once the normalized images are obtained, PPCA is applied on them to extract the features. The low dimension approximation of the data is obtained with the help of the principal components. The approximations obtained for the left and right irises are fused together to obtain the final template. The fusion method used here is the mean method. In this method, the mean of the feature level outcomes, i.e. the reconstructed approximations, is taken.

Two fused templates are matched to check if they have been obtained from the same person or not. In our approach, matching has been performed using the Hamming Distance classifier. The classifier returns a 


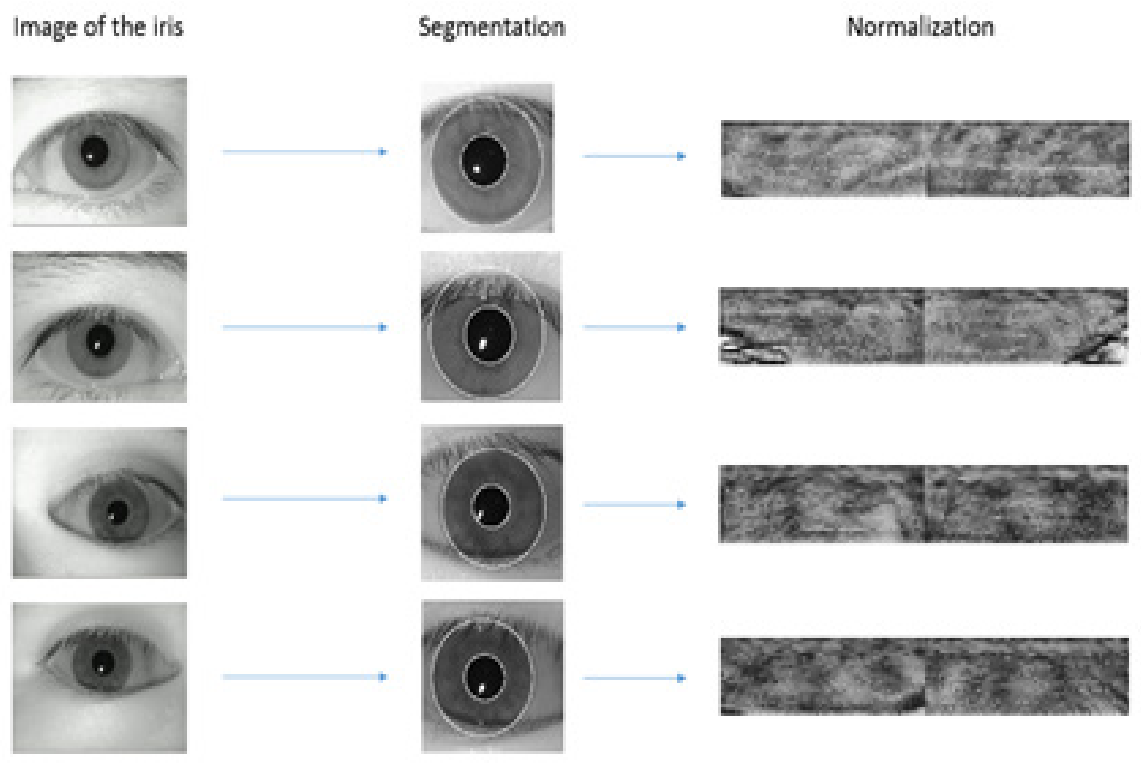

Figure 3. Iris Segmentation and normalization process.

value between 0 and 1 . If the value is less than the threshold (0.32), then the templates match. Else, the templates belong to two different people.

To check for the effectiveness of the proposed approach, we have taken three images each of the left and right irises of eighteen different people randomly from the MMU Iris Dataset (54 sets).

The templates belonging to the same person are matched to check if the desired result, which is a Hamming distance value below the threshold, is obtained or not. After matching all the templates, a recognition rate of $83.3 \%$ (approximated) is obtained. Even with inaccurately segmented images, the recognition rates obtained give a promising result. Table 1 provides brief information about the experimental observations achieved. Total number of subjects used was 18, having 3 left and 3 right eye images each. Total number of feature level fused templates was 54 . Out of the 54 templates experimental results gave 45 true positive matches and 9 false negative matches. Detection rate is identified by using the formula

$$
\frac{T P}{T P+F N} \frac{T P}{T P+F N}
$$

Table 1. Experimental observations

\begin{tabular}{|c|c|c|c|c|}
\hline No. of Subjects & $\begin{array}{c}\text { No. of fused F.V. (left } \\
\text { \& right) }\end{array}$ & $\begin{array}{c}\text { No. of } \\
\text { TN }\end{array}$ & $\begin{array}{c}\text { No. of } \\
\text { FN }\end{array}$ & $\begin{array}{c}\text { Detection Rate } \\
\text { (DR=TP/(TP+FN) }\end{array}$ \\
\hline 18 & 54 & 45 & 9 & $83.3 \%$ \\
\hline
\end{tabular}


True positive occurs when an unknown sample matches a particular subject's fused template stored in database and the match is correct.

False Negative occurs when the sample does not belong to anyone's entry in the database and it in fact belongs to a subject present in the database.

\section{Conclusion}

In this paper, a PPCA based feature selection technique is used to overcome the difficulties faced during segmentation process of non-ideal images. By using PPCA, time taken to remove the effect of noises present in the input image is reduced thus increases the robustness of the system. In order to deal with sensor data noises and spoof attacks, multi-unit feature level fusion technique using mean method of fusion is used. The fused templates are matched using Hamming distance classifier. The recognition rate has been calculated by checking the number of results which are the threshold. The obtained recognition rate is $83.3 \%$ (approximately) even with inaccurately segmented images. The entire work was implemented using MATLAB R2015a.

\section{References}

1. Arun Ross, Anil Jain. Information fusion in biometrics. Pattern Recognition Letters. 2003 Sep; 24(13):2115-25.

2. Alice Nithya A, Lakshmi C. Iris Recognition Techniques: A Literature Survey. International Journal of Applied Engineering Research. 2015 Jul; 10(12):32525-46.

3. Hyun-Ae Park, Kang Ryoung Park. Iris recognition based on score level fusion by using SVM. Pattern Recognition Letters. 2007 Nov; 28(15):2019-28.

4. Jain Jang, Kang Ryoung Park, Jinho Son,Yillbyung Lee. Multi-unit iris recognition system by image check algorithm. Springer Berlin Heidelberg in Biometric Authentication. 2004 Jul, p. 450-57.

5. Banday, Shoaib Amin, Ajaz Hussain Mir, Farida Khursheed. Multi-unit iris biometric fusion using gray level cooccurrence matrix features. International Conference on Advanced Electronic Systems (ICAES). 2013 Sep, p. 225-29.

6. Hunny Mehrotra, Mayank Vatsa, Richa Singh,Banshidhar Majhi. Biometric match score fusion using RVM: A case study in multi-unit iris recognition. IEEE Computer Society Conference in Computer Vision and Pattern Recognition Workshops (CVPRW). 2012 Jun, p. 65-70.

7. John Daugman. How iris recognition works. IEEE Transactions on Circuits and Systems for Video Technology. 2004 Jan;14(1):21-30.

8. John G Daugman. High confidence visual recognition of persons by a test of statistical independence. IEEE Transactions on Pattern Analysis and Machine Intelligence. 1993 Nov; 15(11):1148-61.

9. Ilin A, Raiko T. Practical Approaches to Principal Component Analysis in the Presence of Missing Values. Journal of Machine Learning Research (JMLR). 2010 Jan; 11:1957-2000.

10. Roweis S. EM Algorithms for PCA and SPCA. In: Proceedings of the 1997 Conference on Advances in Neural Information Processing Systems.1998, p. 626-32.

11. Tipping ME, Bishop CM. Probabilistic Principal Component Analysis. Journal of the Royal Statistical Society. Series B (Statistical Methodology). 1999; 61(3):611-22.

12. Iris recognition. https://en.wikipedia.org/wiki/Iris_recognition.Date Accessed: 18/07/2016. 